\title{
Torin2 targets dysregulated pathways in anaplastic thyroid cancer and inhibits tumor growth and metastasis
}

\author{
Samira M. Sadowski ${ }^{1}$, Myriem Boufraqech ${ }^{1}$, Lisa Zhang ${ }^{1}$, Amit Mehta ${ }^{2}$, Payal \\ Kapur $^{3}$, Yaqin Zhang ${ }^{4}$, Zhuyin $\mathrm{Li}^{4}$, Min Shen ${ }^{4}$ and Electron Kebebew ${ }^{1}$ \\ ${ }^{1}$ Endocrine Oncology Branch, National Cancer Institute, National Institutes of Health, Bethesda, MD, USA \\ 2 Geisel School of Medicine at Dartmouth, Hanover, NH, USA \\ ${ }^{3}$ Department of Pathology, University of Texas Southwestern Medical Center, Dallas, TX, USA \\ ${ }^{4}$ Division of Discovery Innovation, National Center for Advancing Translational Sciences, National Institutes of Health, \\ Bethesda, MD, USA \\ Correspondence to: Samira M. Sadowski, email: samira.sadowskiveuthey@nih.gov \\ Keywords: torin2, mTORC 1 inhibitor, mTOR, anaplastic thyroid cancer, quantitative high-troughput screening \\ Received: February 20, 2015 \\ Accepted: March 26, 2015 \\ Published: April 14, 2015
}

This is an open-access article distributed under the terms of the Creative Commons Attribution License, which permits unrestricted use, distribution, and reproduction in any medium, provided the original author and source are credited.

\section{ABSTRACT}

Anaplastic thyroid cancer (ATC) is rare but it is one of the most lethal human malignancies with no effective therapy. There is a pressing need to identify new therapeutic agents for ATC. We performed quantitative high-throughput screening (qHTS) in ATC cell lines using a compound library of 3,282 drugs. qHTS identified 100 pan-active agents. Enrichment analysis of qHTS data showed drugs targeting mTOR were one of the most active drug categories, and Torin2 showed the highest efficacy. We found mTOR to be upregulated in ATC. Treatment of multiple ATC cell lines with Torin 2 showed significant dose-dependent inhibition of cellular proliferation with caspase-dependent apoptosis and G1/S phase arrest. Torin 2 inhibited cellular migration and inhibited the phosphorylation of key effectors of the mTOR-pathway (AKT, 4E-BP1 and 70S6K), as well as claspin and survivin expression, regulators of cell cycle and apoptosis. In our in vivo mouse model of metastatic ATC, Torin2 inhibited tumor growth and metastasis and significantly prolonged overall survival. Our findings suggest that Torin 2 is a promising agent for ATC therapy and that it effectively targets upregulated pathways in human ATC.

\section{INTRODUCTION}

Anaplastic thyroid cancer (ATC) is one of the most deadly solid human malignancies. It represents less than $2 \%$ of all thyroid cancers, has an annual incidence of 1-2 cases per million population per year but accounts for nearly one-third of thyroid cancer deaths $[1,2]$. The median survival of patients with ATC is less than 6 month, and $90 \%$ of patients with ATC present with unresectable locally advanced or distant metastatic disease at time of diagnosis [3, 4]. There is currently no standard or effective therapy for ATC and the survival of patients with ATC has not improved in over 6 decades [3,5]. Thus, there is an unmet need to identify effective agents for the treatment of ATC.

Drug discovery and development can be costly and take a long time to identify a clinically effective agent.
For example, it has been estimated that to bring a single drug to market takes an average of 15 years and costs approximately $\$ 800$ million [6]. Therefore, quantitative high-throughput screening (qHTS) of existing drugs has emerged as an attractive alternative, especially for orphan cancers such as ATC [7-9]. Screening a drug library has several advantages, which include knowledge of the drugs pharmacokinetic, pharmacodynamics and toxicity profile. This information could lead to a more efficient repurposing of drugs for rare cancers. Another important advantage of screening a Food and Drug Administration (FDA) approved drug library with known mechanism of action, is the ability to determine whether compound(s) found to be active on qHTS can target molecular pathways that are altered in human malignancies.

In this study, we performed qHTS in multiple ATC cell lines, and identified active compounds that were 
enriched for drugs targeting the mTOR pathway. We then demonstrate that the mTOR pathway is upregulated in ATC with validation of the potent anticancer effects of an irreversible potent mTOR inhibitor, Torin2, in vitro and in vivo. Furthermore, we show Torin 2 mediates caspasedependent apoptosis while reducing the anti-apoptosis protein survivin, which is upregulated in ATC. Lastly, Torin2 also induced G1/S phase arrest in ATC cells resulting in decreased claspin protein levels, a protein required for DNA replication.

\section{RESULTS}

\section{Quantitative high-throughput screening identifies mTOR inhibitors are active in ATC cells}

We identified 100 active agents in the three ATC cell lines screened. Enrichment analysis by mode of drug action showed mTOR inhibitors to be one of the highest-ranking drug categories. Torin2 was the most

\begin{tabular}{|c|c|c|c|}
\cline { 2 - 4 } \multicolumn{1}{c}{} & \multicolumn{3}{c}{ Cell line } \\
\hline Drug Name & 8505 c & SW 1736 & C643 \\
\hline Torin-2 & & & \\
\hline PF-05212384;PKI-587 & & & \\
\hline $\begin{array}{c}\text { GDC-0980;R-7422/ } \\
\text { RG-7422 }\end{array}$ & & & \\
\hline OSI-027 & & & \\
\hline AZD-8055 & & \\
\hline $\begin{array}{c}\text { Everolimus;Afinitor; } \\
\text { NVP-RAD-001 }\end{array}$ & & \\
\hline Torin-1 & & \\
\hline KU-0063794 \\
\hline $\begin{array}{c}\text { Salirasib;KD-032/ } \\
\text { ONO-7056 }\end{array}$
\end{tabular}

Maximum inhibition $-130 \%$

Inactive $0 \%$ active agent of nine mTOR inhibitors in all three ATC cell lines screened based on efficacy (maximum response $>100 \%$ ), best dose-response curve class, and the lowest half-maximum inhibitory concentration $\left(\mathrm{IC}_{50}\right)(\leq 0.8 \mu \mathrm{M})$ (Figure 1A). Although there is no effective chemotherapy agent for ATC, agents such as docetaxel and doxorubicin have been used as monotherapy or in combination [10-12]. Thus, we compared Torin 2 activity in the three ATC cell lines to both agents and found Torin 2 had better activity (maximum response and lower $\mathrm{IC}_{50}$ ) than docetaxel and doxorubicin (Figure 1B).

\section{Torin 2 inhibits cellular proliferation in ATC cell lines, increases caspase activity and decreases cellular migration}

In vitro studies using Torin 2 in seven ATC cell lines showed a significant dose-dependent inhibition of cellular proliferation (Figure 2A). We found high concentrations of Torin 2 were cytotoxic. Thus, we next asked whether Torin 2 induced apoptosis. We found Torin 2 increased
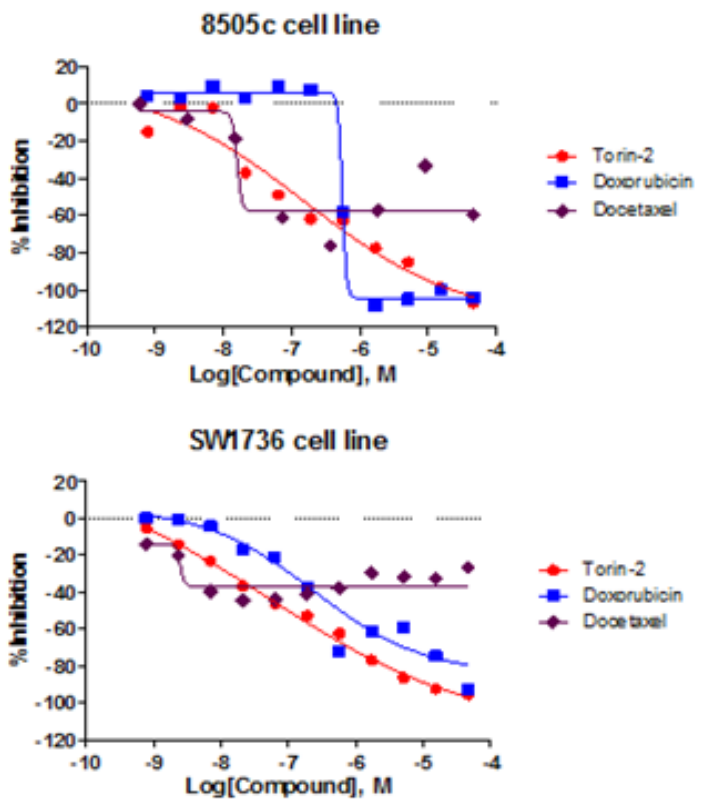

A

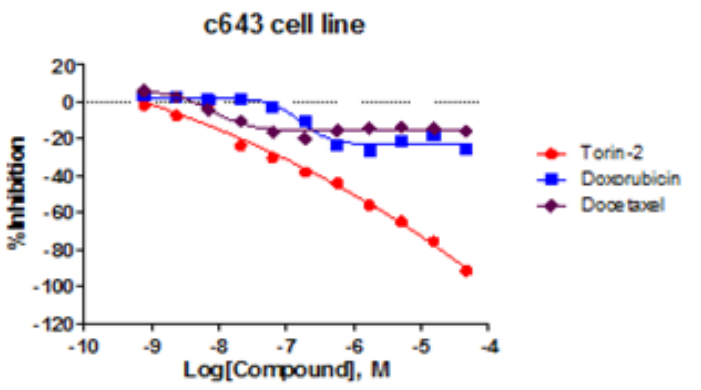

Figure 1: Results of qHTS in ATC cell lines. A. Heatmap of efficacy analysis of drugs analyzed by qHTS in three ATC cell lines (8505c, SW1736 and C643), with maximum response (inhibition). B. Dose-response curves of Torin2, docetaxel and doxorubicin in ATC cell lines. The $\mathrm{Y}$-axis shows percent inhibition and the $\mathrm{X}$-axis shows the drug concentration. 
caspase 3/7 activity, increased the number of cells in G1 and decreased the number of cells in S-phase (Figure 2B2C), which is consistent with the effect on apoptosis [13].

To investigate the mechanism of how Torin2 induced apoptosis and G1/S-phase arrest, we analyzed the expression level of apoptosis-related proteins with an antibody array. We found that Torin 2 reduced claspin,
HIF- $1 \alpha$ and survivin levels in a dose dependent fashion in all three cell lines, as shown in Figure 2D. Torin2 had a dose-dependent effect on survivin protein levels (Figure 2E).

We next investigated whether Torin2 had an effect on cellular migration as ATC is highly invasive and the mTOR pathway has been implicated in regulating cellular
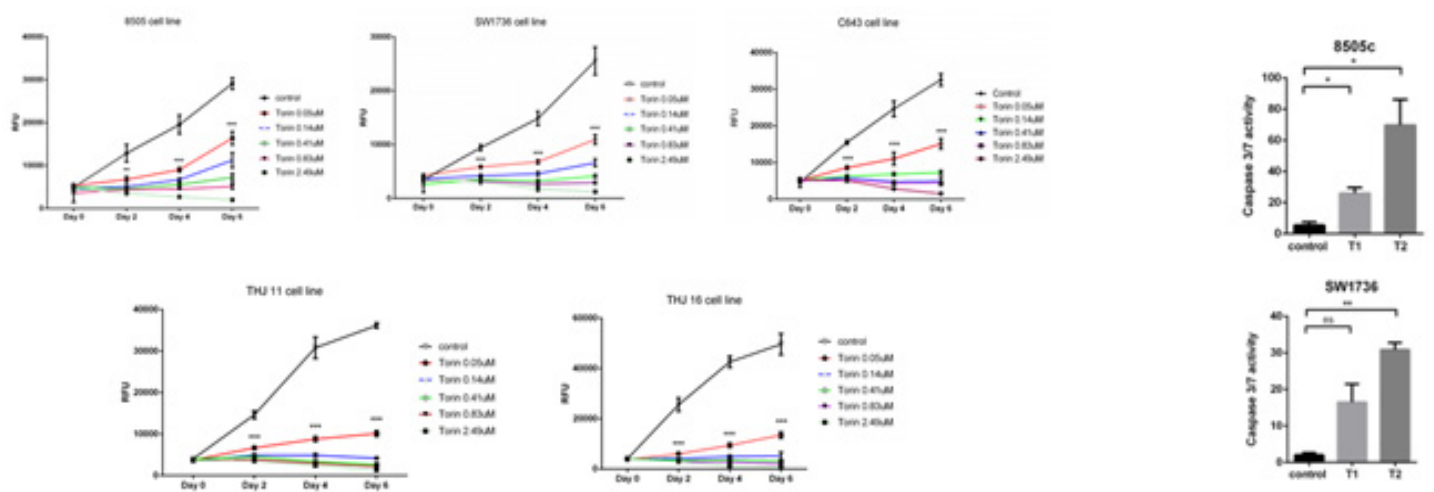

A
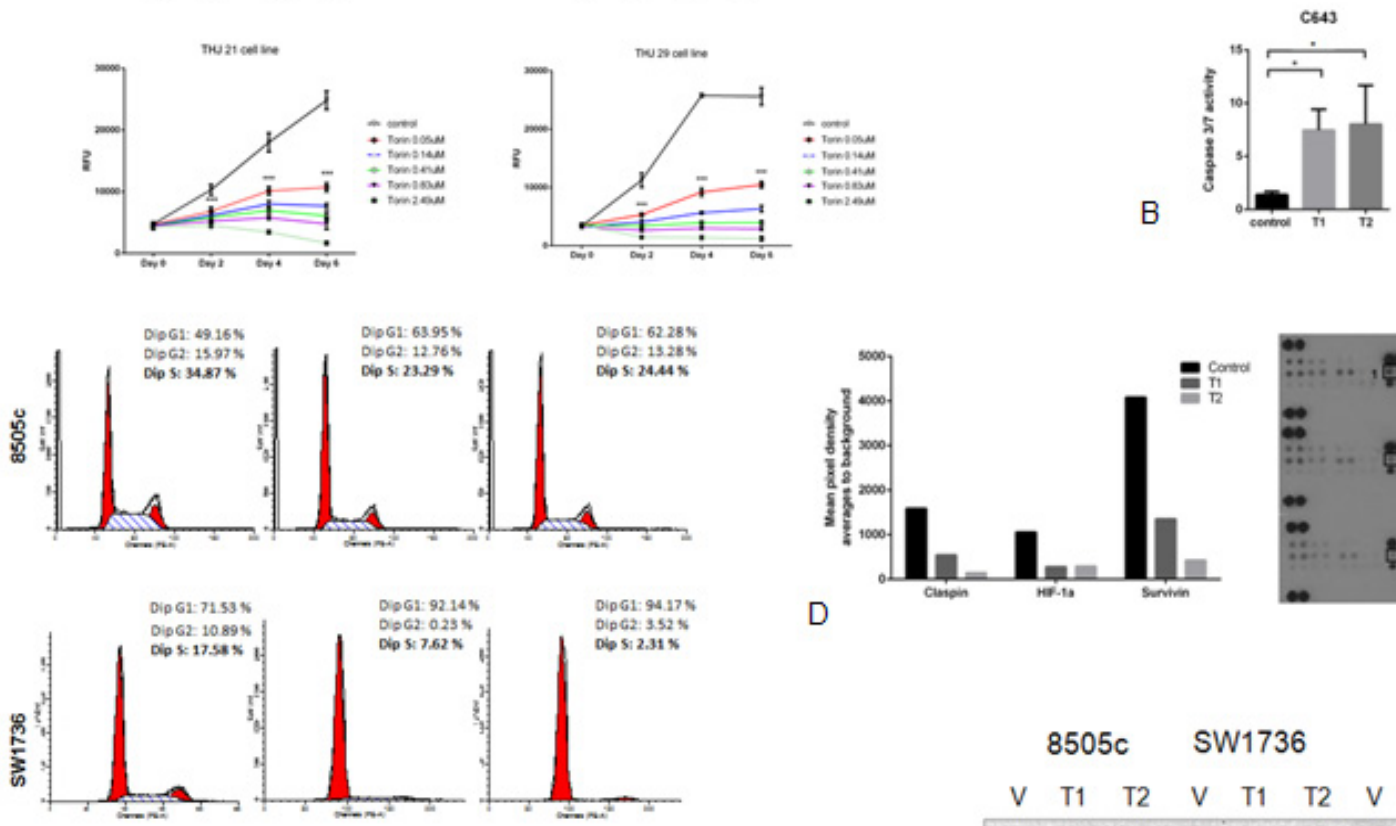

D
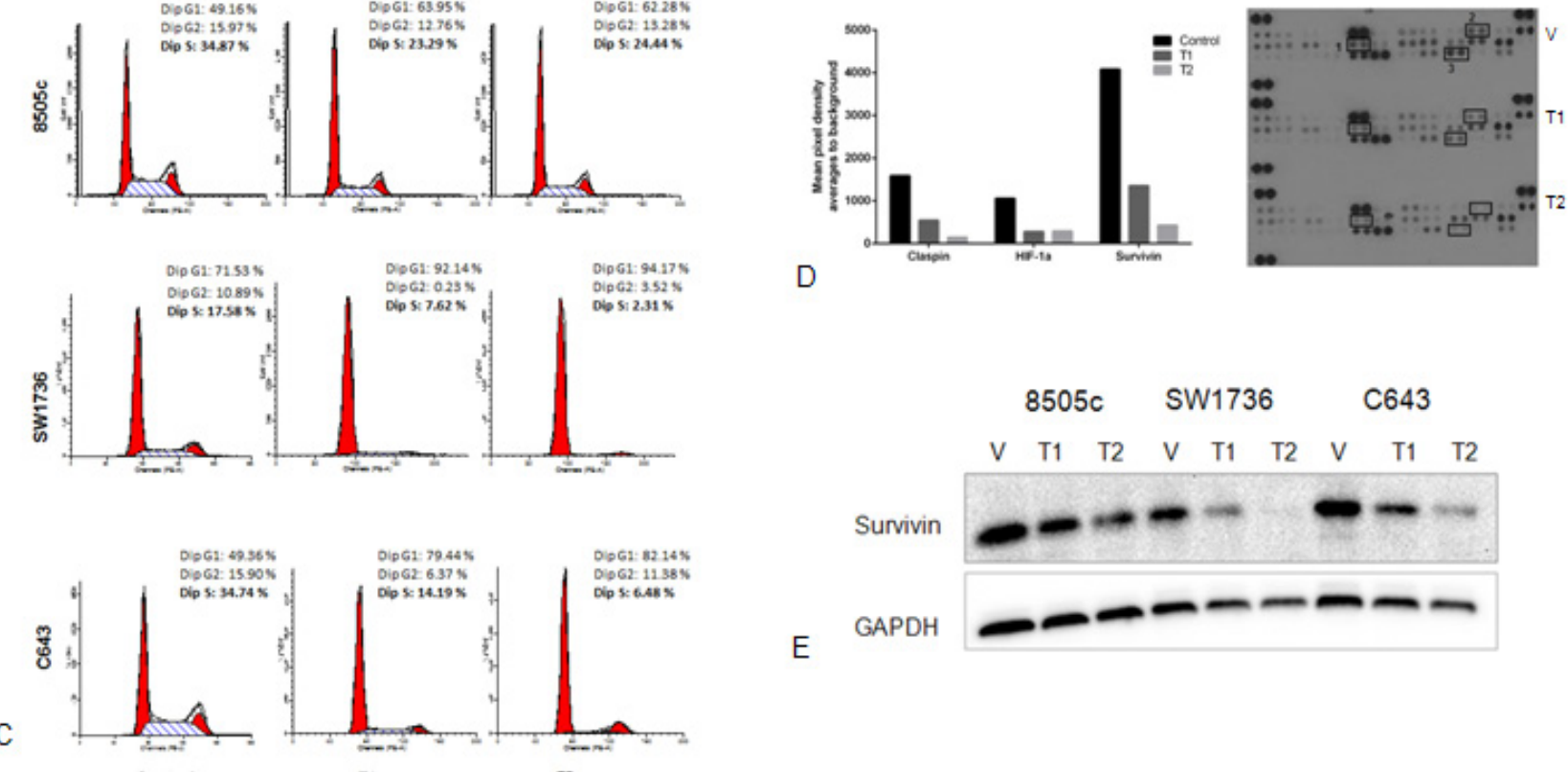

Figure 2: Effect of Torin2 on cellular proliferation, caspase activity and cell cycle in ATC cell lines. A. Torin2 inhibits cellular proliferation in ATC cell lines. Cells were treated with Torin2 ( 0 to $2.49 \mu \mathrm{M})$ diluted in DMSO (control vehicle) for 6 days. RFU: Relative Fluorescence Unit. ${ }^{*} p<0.01,{ }^{*} p<0.001, * * * p<0.0001$. B. Torin 2 increases caspase $3 / 7$ activity in ATC cell lines. CaspaseGlo 3/7 assay was performed in three ATC cell lines after 48 hours of treatment using the two lowest concentrations of Torin2 used in the proliferation assays (Figure $2 \mathrm{~A}, \mathrm{~T} 1=0.05 \mu \mathrm{M}$ and $\mathrm{T} 2=0.14 \mu \mathrm{M}$ ) ${ }^{*} p<0.05,{ }^{* *} p<0.005$, ns $=$ non-significant. C. Cell cycle analysis was performed after 24 hours of treatment of Torin2. T1 $=0.05 \mu \mathrm{M}$ and $\mathrm{T} 2=0.14 \mu \mathrm{M}$. D. Effect of Torin2 on apoptosis-related proteins. ATC cells were treated for 48 hours using DMSO as control and Torin2 at T1 $=0.05 \mu \mathrm{M}$ and T2 $=0.14 \mu \mathrm{M}$. A representative graph with corresponding scanned images is shown in Figure 2D for cell line C643. 1: HIF-1 $\alpha$, 2: Claspin, 3: Survivin. E. Western blot analysis of survivin after 48 hours of treatment with Torin 2 at $\mathrm{T} 1=0.05 \mu \mathrm{M}$ and $\mathrm{T} 2=0.14 \mu \mathrm{M}$. 
migration and epithelial-mesenchymal-transition (EMT), a feature omnipresent in ATC $[14,15]$. Torin2 significantly inhibited cellular migration in 2 of 3 ATC cell lines, with a trend in $8505 \mathrm{c}$ cells when compared to control (Figure $3 \mathrm{~A})$. Given this effect on cellular migration, we evaluated whether Torin2 had an effect on proteins known to mediate EMT and found no significant effect on Vimentin, CD44 and N-cadherin protein levels (Figure $3 \mathrm{~B}$ ).

\section{Torin2 inhibits mTORC1 and the phosphorylation of mTOR-pathway related proteins}

We next confirmed the inhibition of mTOR by Torin2 in ATC cell lines. Torin 2 decreased phosphorylation of mTOR on Ser 2448, which is specific to the mTORC1 site and total mTOR levels (Figure 4A). Torin2 also decreased phosphorylation of AKT Ser473 and total AKT levels in a dose dependent fashion in all three ATC cell lines (Figure 4A). We next analyzed the downstream effectors of mTORC1, phospho-proteins 4E-BP1 and S6K [16, 17]. Torin2 showed a dose-dependent inhibition of phospho-4E-BP1 and S6K, and total 4E-BP1 in all 3 ATC cell lines; as well as a dose-dependent inhibition of phospho-PRAS40, which is a component and substrate of mTORC1 and a substrate of AKT (Figure 4B) [18].
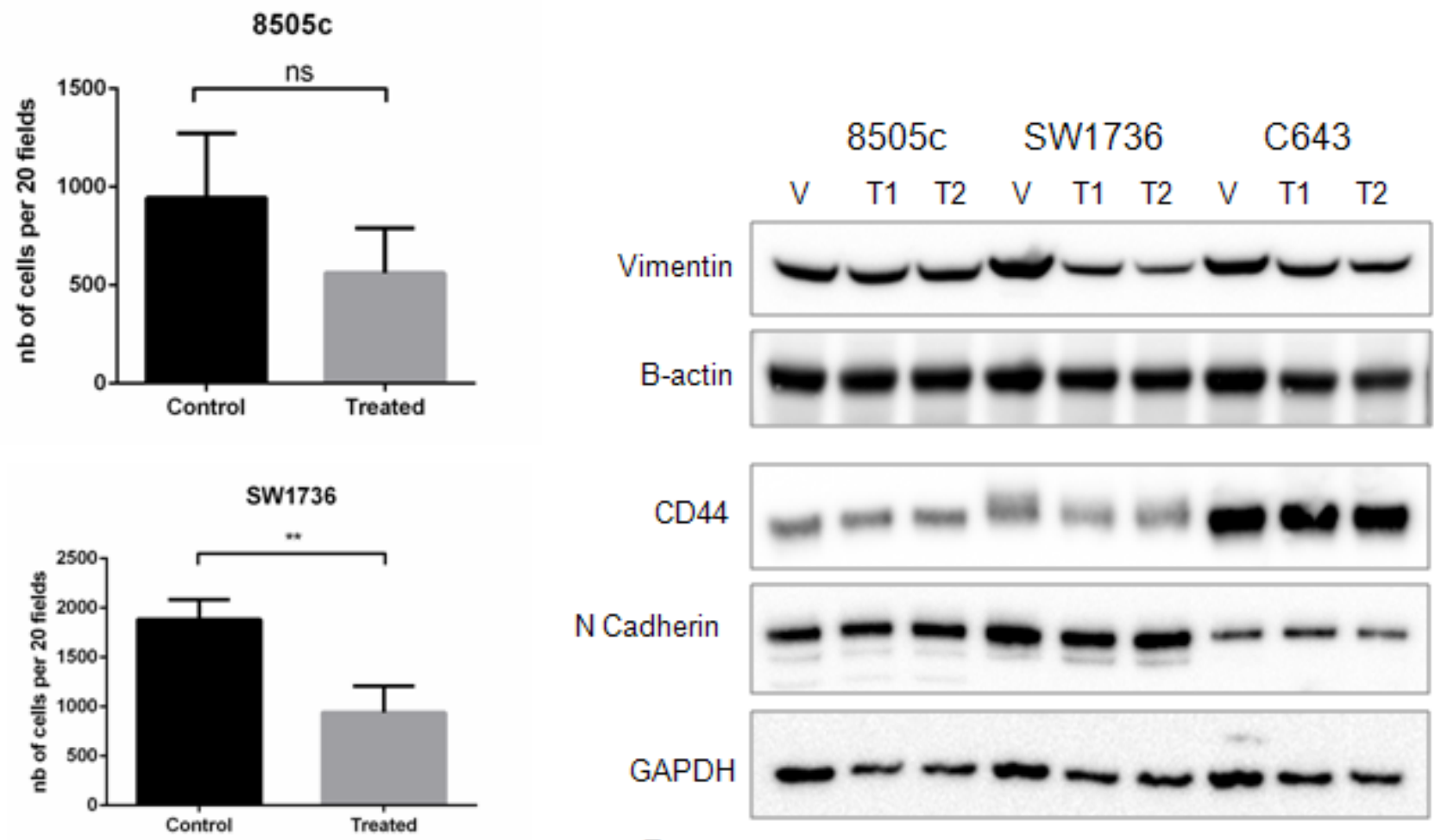

B

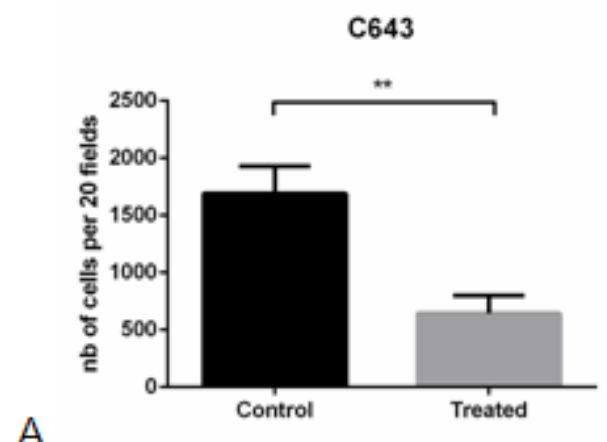

Figure 3: Effect of Torin2 on cellular migration and EMT marker expression. A. Torin2 inhibits cellular migration. A transwell chamber assay was used to measure cellular migration with and without Torin2 treatment for 48 hours at $0.14 \mu \mathrm{M}$. * $p<0.05, * * p<0.005$, $\mathrm{ns}=$ non-significant. $\mathrm{nb}$ on $\mathrm{y}$-axis $=$ number of cells. $\mathbf{B}$. Western blots analysis of Epithelial-Mesenchymal-Transition (EMT) markers after 48 hours of treatment did not affect Vimentin, CD44 and N-cadherin protein levels. T1 $=0.05 \mu \mathrm{M}$ and $\mathrm{T} 2=0.14 \mu \mathrm{M}$. 


\section{In vivo Torin2 inhibits tumor growth and metastases}

Our in vitro data showed that Torin2 treatment inhibits ATC cellular proliferation, induces caspasedependent apoptosis, decreased cellular migration, and effectively inhibits mTOR and mTORC1-related effectors of the mTOR pathway. Therefore, we investigated the effect of Torin 2 treatment in a metastatic mouse model of ATC, which mimics the heavy tumor burden seen in human metastatic ATC [19]. We first evaluated if pretreatment of mice could reduce the rate of metastasis given the effects on cellular migration we observed in vitro. Torin 2 significantly reduced ATC metastasis in mice pretreated with Torin2 (Figure 5A-5B). We then treated mice with established metastasis with Torin2. We found decreased growth and metastases with Torin2 treatment (Figure 5C-5F). The tumor burden was approximately one-third in the treated mice, with fewer mice developing diffuse metastases (liver and bone) with treatment (16\% versus 67\%) (Figure 5C-5F). Torin 2 treatment in mice with established metastasis was also associated with longer survival time, median 1 week longer, $(p<0.005)$ (Figure 5G). We observed no toxicity with Torin2. Weekly weights were stable in both groups until week 4 , and then decreased in the control group with increasing tumor burden.

\section{Torin2 targets are upregulated in ATC}

Given that Torin2 had potent in vitro and in vivo effects in ATC, we next determined if the proteins targeted by this agent are dysregulated in ATC. Using human thyroid tissue arrays, we compared the staining of phospho-mTOR and mTOR in ATC versus normal and benign thyroid tissues. For mTOR, the H-score was significantly higher in ATC samples (the average readings of the duplicate sections showed an H-score of 1.6 in the normal and benign samples versus 147 in ATC samples, $p<0.05$ ) (Figure 6A-6B). Additionally, we found Torin2 reduced survivin levels in vitro and therefore analyzed the expression of this protein in ATC. We found survivin protein expression to be positive in ATC and papillary thyroid cancer compared to normal thyroid tissue samples (Figure 6A), consistent with evidence that survivin overexpression is associated with progression to poorly differentiated thyroid cancer [20] and reduced survival in colorectal cancer [21]. These findings suggest that the targets of Torin2 are upregulated in ATC making it a rationale targeted agent for ATC. Further, we analyzed
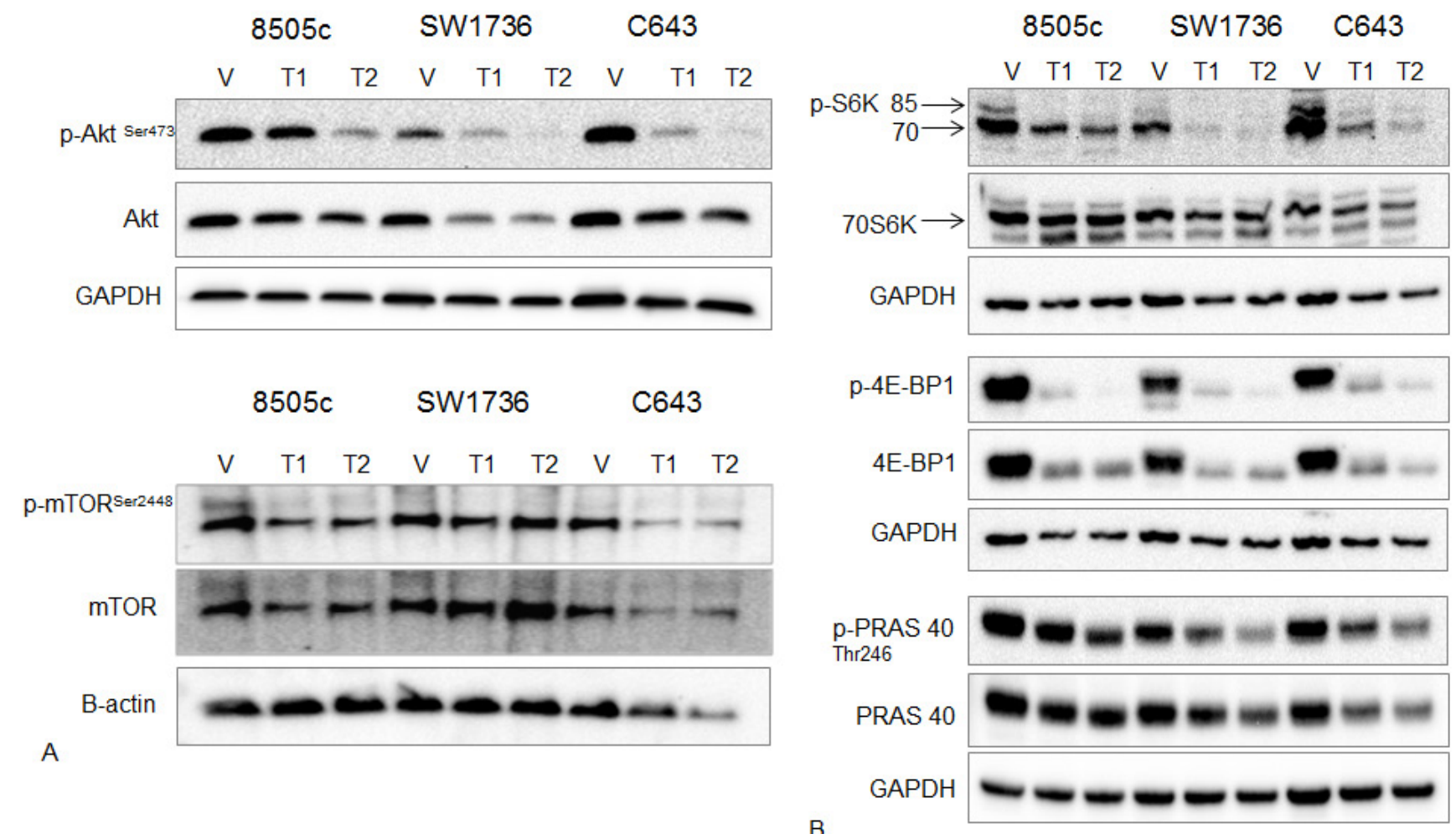

Figure 4: Effect of Torin2 on mTOR and mTOR-related protein expression and phosphorylation. A. Western blot analysis of AKT, phospho-AKT ${ }^{\text {Ser473 }}, \mathrm{mTOR}^{\text {Ser2448 }}$ (mTORC1 site) and total mTOR. ATC cells were treated with Torin2 for 48 hours at $\mathrm{T} 1=0.05$ $\mu \mathrm{M}$ and $\mathrm{T} 2=0.14 \mu \mathrm{M}$. Beta-actin was used as a loading control for the mTOR blot because of the higher molecular weight. B. Western blot analysis of downstream targets of mTOR: phospho-S6K (p-S6K), total 70S6K, phospho-4E-BP1 (p-4E-BP1), total 4E-BP1, phospho PRAS40 (p-PRAS40) and total PRAS40 with Torin2 treatment for 48 hours in ATC cells at T1 $=0.05 \mu \mathrm{M}$ and T2 $=0.14 \mu \mathrm{M}$. 
tumor samples from mice responding to Torin2 treatment and found reduced phospho-4E-BP1 staining, as well as reduced staining for survivin (reduced nuclear staining) and HIF-1 $\alpha$, a marker of aggressive cancer (Figure 6C) $[22,23]$.

\section{DISCUSSION}

We performed qHTS in ATC cell lines and identified mTOR inhibitors to be one of the most active drug categories. Torin2 was the most active compound in ATC cells and effectively inhibited cellular proliferation, induced increased caspase activity and S-phase arrest,
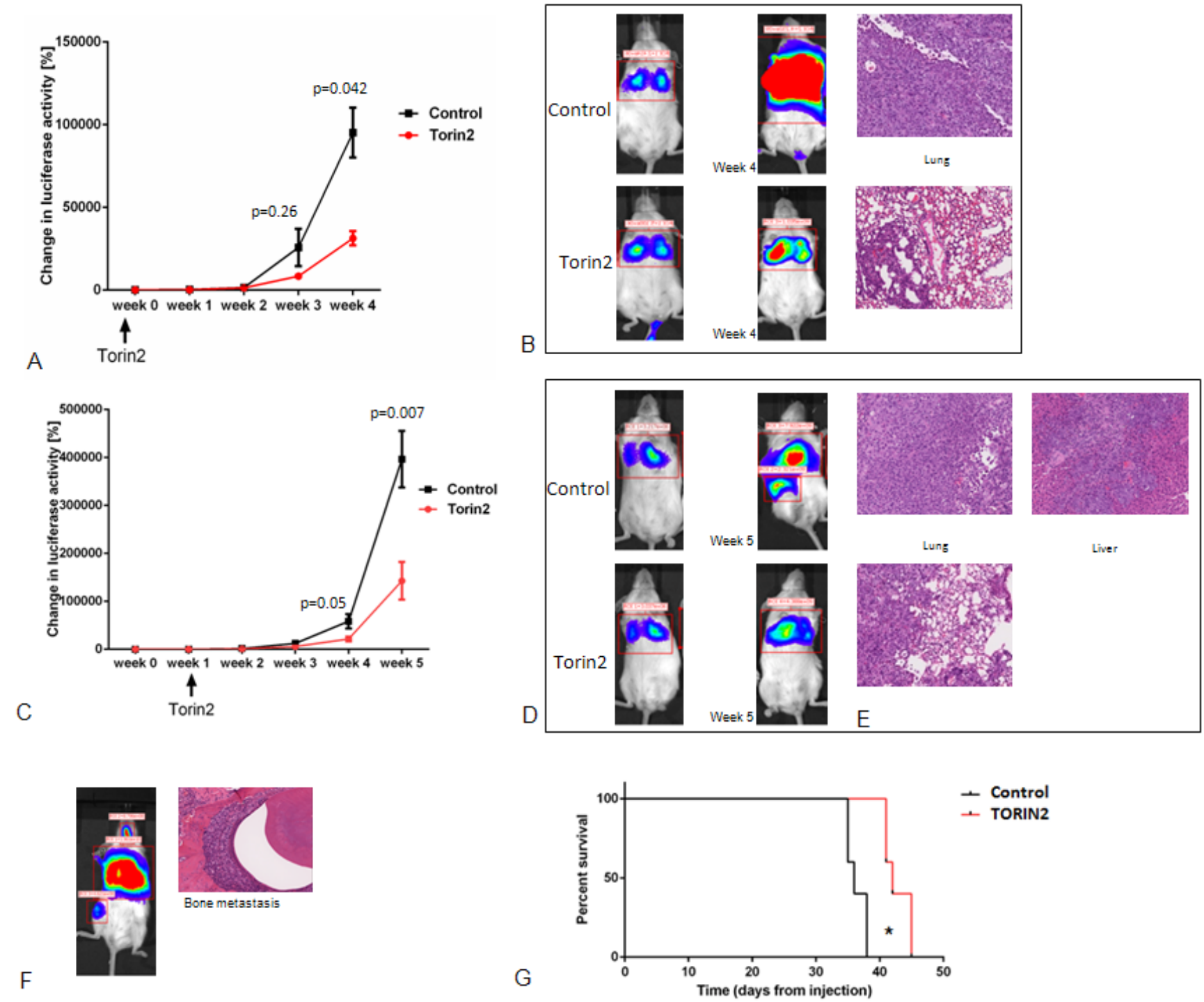

Figure 5: Effect of Torin2 on tumor growth and metastases in vivo. A. and B. Pretreatment of mice with Torin2 decreases metastasis and tumor growth. Mice $(n=6)$ were treated by oral gavage 24 hours with either Torin $220 \mathrm{mg} / \mathrm{kg}$ or Captisol before tumor engraftment of $8505 \mathrm{c}$ luc cells via the tail vein. A. Y axis in A indicates total change in luciferase activity per mouse as measured from the day of tumor cell injection and weekly. $X$ axis shows the week after engraftment with the treatment timeline below. Results of lung tumor intensity, measured as change in luciferase activity in \% per mouse (at 4 weeks $p<0.05$, mean \pm SEM). B. Representative whole mouse images of treated and control mice at 4 weeks after cell engraftment as well as representative H\&E section of lung metastasis. C. and D. Torin 2 inhibit growth and metastases in vivo. Mice $(n=12)$ were engrafted with $8505 \mathrm{c}$ luc cells via the tail vein and given daily oral gavage treatment with Torin $20 \mathrm{mg} / \mathrm{kg}$ or Captisol at 1 week after the establishment of metastasis. C. Y axis indicates total change in luciferase activity in \% per mouse as measured the day of tumor cell injection and then weekly (week $4, p=0.05$ and week $5, p=0.007$, mean \pm SEM). D. Representative images of control and treated mouse with signal intensity for lung metastasis and at other sites of metastasis. E. Representative H\&E images of Torin2 treated mice compared to the control mice (20X). A representative example of IVS image for signal intensity and the corresponding H\&E for bone metastasis in a non-treated mouse is shown in F. G. Kaplan Meier survival curve with and without Torin 2 treatment ( $n=6$ in each group). Median survival of 42 days in the Torin 2 treated mice versus 36 days in the control mice $(p=0.0025$, Mantel Cox log rank test). 
and inhibited cellular migration in vitro. In addition to inhibiting mTOR and the downstream effectors of the mTOR pathway (4E-BP1 and S6K), Torin2 inhibited the phosphorylation of AKT, an important signaling (PI3K/ AKT/mTOR)-pathway in ATC and a variety of cancers [24]. In vivo, Torin 2 inhibited growth and metastasis. Further, we show Torin 2 to be more active than current therapies such as docetaxel and doxorubicin, and demonstrate that the targets of Torin 2 are upregulated in ATC making it an attractive targeted therapy for ATC.

The mTOR-pathway controls cell survival and cell growth and is involved in multiple cancers and has been actively investigated as a target for cancer therapies [25, 26]. Several studies have investigated the mTOR-pathway in ATC. Papewalis and colleagues showed that everolimus inhibits the growth of ATC cell lines in a dose-dependent fashion [27] and Liu and associates have shown that phospho-mTOR is activated in ATC [28] consistent with our results. However, one of the shortcomings of using everolimus and other rapalogs to target the PI3K/ AKT/ mTOR-pathway is the development of resistance with long-term treatment as a result of reactivation of AKT and mTORC2 as these drugs target only mTORC1 [26, 29, 30]. More promising results have been observed in lung cancer and renal cell carcinoma models if the mTOR-pathway is targeted by inhibiting both AKT and mTOR $[31,32]$. To overcome this limitation of AKT reactivation with everolimus and other rapalogs treatment, new second generation, ATP-competitive drugs have been developed that target both mTOR-complexes, mTORC1 and mTORC2, such as Torin2. Liu et al. [32] showed that Torin 2 inhibits AKT and mTORC1, overcoming the feedback reactivation of AKT due to long term mTORC1 treatment, but it was effective in an in vivo lung tumor model only when combined with a MEK inhibitor. Simioni et al. [33] showed promising dual mTORC1 and mTORC2 inhibition with Torin2 in B-precursor acute lymphoblastic leukemia. Similar results have been reported in papillary
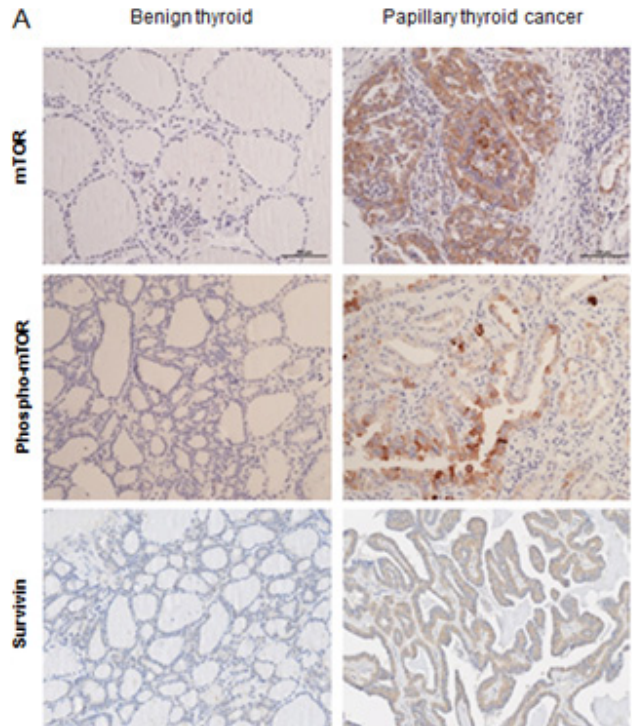

C
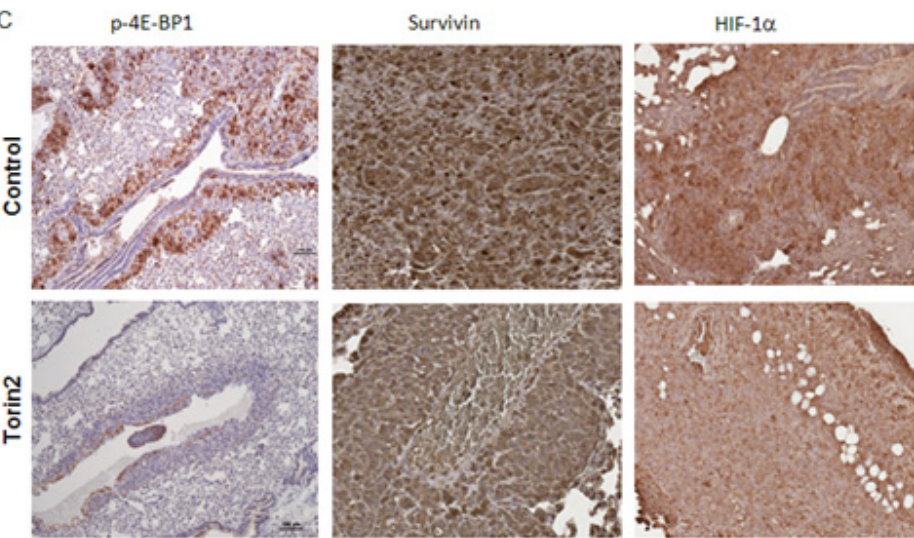

B
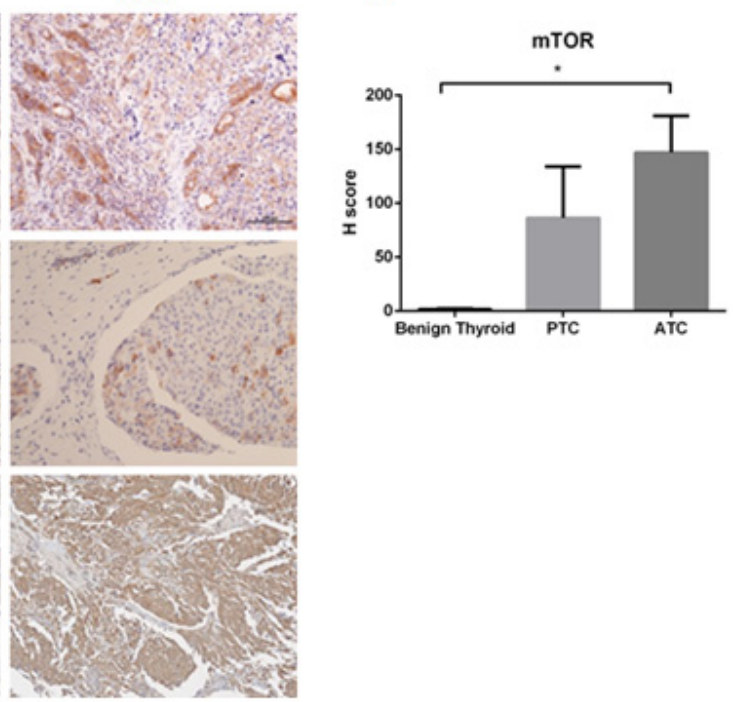

Figure 6: Torin2 targets are dysregulated in ATC. A. Representative images of phospho-mTOR, mTOR and survivin staining in benign thyroid tissue, papillary thyroid cancer and ATC. B. Graph of H score of mTOR staining in 6 ATC tissues ( 1 was necrotic) $(=12$ sections), 6 PTC (=12 sections) versus 6 normal and 6 benign (follicular adenoma) (=24 sections) thyroid tissues, $p=0.013(*)$, mean \pm SEM. C. Representative images of whole mouse lung metastases at 4 weeks after cell engraftment with phospho-4E-BP1 staining. Representative images of survivin (reduced nuclear staining in the Torin 2 group) and HIF-1 $\alpha$ staining. Images at 20-100X. 
thyroid cancer using a tumor flank xenograft model [34]. Lastly, a recent clinical report of a patient with metastatic ATC enrolled in a Phase II trial of everolimus therapy, showed resistance after an extraordinary 18 months of response. Whole-exome sequencing of pretreatment and drug resistant tumors revealed a mutation in mTOR that conferred resistance to allosteric mTOR inhibition (everolimus), but the mutation remained sensitive to a direct ATP-competitive mTOR kinase inhibitor such as Torin1 [35].

To our knowledge, this is the first study that shows Torin2 is active in ATC cells and in an in vivo mouse model of ATC that recapitulates the heavy tumor burden seen in patients with ATC [19]. We also show for the first time that Torin 2 treatment in ATC cells results in lower claspin and survivin levels in ATC cells consistent with its effect on G1/S phase arrest and apoptosis, respectively $[20,36,37]$. Furthermore, Torin2 treatment in vivo was associated with reduced phospho-4E-BP1, HIF-1 $\alpha$ and survivin protein expression in ATC responsive tumor samples. These data taken together show Torin2 to be potent in ATC suggesting it would be a good targeted therapeutic agent in patients with ATC.

In ATC, TP53 inactivating mutations are most common, occurring in $42-55 \%$ of cases, followed by mutations in genes involved in the $\mathrm{PI} 3 \mathrm{~K} / \mathrm{AKT} / \mathrm{mTOR}$, MAPK and WNT signaling pathways, mutations in $R A S$ and $B R A F(25 \%)[38,39]$, and mutations in PIK3CA and PTEN, that occur in $17-23 \%$ and $12 \%$, respectively, of ATC cases [38, 40]. Anti-BRAFV600E therapy has shown good activity in BRAF mutant cells in vitro and in vivo [41-43]. We found that Torin2 was effective in all cell lines harboring a similar mutation profile $(B R A F$ V600E, TP53, HRAS, PTEN, KRAS, RB, and PI3KCA) as observed in human ATC tumor samples. This suggests that the activity of Torin 2 is independent of the presence of driver mutations, which may activate the alternate MAPK pathway. We also identified claspin and survivin as new targets, which are dysregulated in ATC and that are reduced with Torin2 treatment in ATC cells. Survivin is upregulated in human ATC samples and is associated with more aggressive disease in differentiated thyroid cancer $[20,37]$. These findings further support that Torin 2 may be effective in patients with locally advanced and metastatic ATC.

In summary, we show that the mTOR-pathway is upregulated in ATC and that mTOR inhibitors are potent in ATC cell lines in vitro and in vivo. Torin 2 effectively inhibits mTOR and mTOR-pathway effectors at multiple levels, and dysregulated protein targets in ATC such as survivin. Our findings taken together show that Torin 2 is a good candidate drug for ATC treatment and should be considered for a clinical trial in patients with ATC.

\section{MATERIALS AND METHODS}

\section{Quantitative high-throughput screening (qHTS) and compound library}

The compound library consists of 3,282 small molecules that are either drugs that have been approved for human or animal use by the United States FDA or drug candidates that are currently in investigational use. Cell viability after drug treatment was measured using a luciferase-coupled ATP quantification assay (CellTiterGlo ${ }^{\circledR}$, Promega, Madison, WI) in three ATC cell lines: 8505c, C643, and SW1736. qHTS was performed as previously described [7, 9]. To determine compound activity in the qHTS assay, the titration-response data was plotted and modeled by a four parameter logistic fit yielding half maximum inhibitory concentration $\left(\mathrm{IC}_{50}\right)$ and efficacy (maximal response) values. Raw plate reads for each titration point were normalized relative to a positive control defined as 100\% inhibition (Tetraoctylammonium bromide, 100\% inhibition) and DMSO only wells (defined as $0 \%$ inhibition). To assess the drug categories that were active in 8505c, C643, and SW1736 ATC cells, we performed enrichment analysis by the drug mode of action. The enrichment score was defined as the ratio of number of active drugs to the total of number tested drugs in that drug category (mode of action).

\section{mTOR inhibitor Torin2}

Torin 2 was purchased from Selleckchem (Houston, $\mathrm{TX}$ ) for in vitro and in vivo experiments. The drug was diluted in DMSO for in vitro studies and in Captisol ${ }^{\circledR}$ (Ligand Pharmaceuticals, Inc., La Jolla, CA) for animal studies.

\section{Cell lines}

Human ATC cell lines, 8505c (purchased from the European Collection of Cell Cultures, Salisbury UK), C643 and SW1736 (purchased from CLS Cell Lines Service GmbH, Germany), and THJ-11T, THJ-16T, THJ-21T and THJ-29T (kindly provided by Dr. John A. Copland III, Mayo Clinic, Jacksonville, FA) were maintained in Dulbecco's modified Eagle's medium (DMEM) supplemented with $10 \%$ fetal calf serum (FCS), penicillin $(100 \mathrm{U} / \mathrm{mL})$, streptomycin $(100 \mu \mathrm{g} /$ $\mathrm{mL})$, fungizone $(250 \mathrm{ng} / \mathrm{mL})$, TSH (10 IU/L), and insulin $(10 \mu \mathrm{g} / \mathrm{mL})$ in $5 \% \mathrm{CO} 2$ atmosphere at $37^{\circ} \mathrm{C}$. All cell lines were authenticated by short tandem repeat profiling. The most relevant mutations profile of the ATC cell lines are 8505c (BRAF V600E, TP53), C643 (HRAS, TP53, PTEN), SW1736 (BRAF V600E, TP53, 
and $P I K 3 C B)$, THJ-11T (KRAS), THJ-16T (TP53, RB, and PI3KCA), THJ-21T (TP53, RB, and BRAF V600E), and THJ-29T $(R B)$ [44]. For 8505c, C643 and SW1736 targeted sequencing using the Ion Torrent TargetSeq platform (Life Technologies, Grand Island, NY) was done for known tumor suppressor and oncogenes.

\section{Cell proliferation, apoptosis and cell cycle assays}

Cells were plated in 96-well plates and treated with Torin2 or vehicle control (DMSO) and the CyQUANT assay (Life Technologies, Grand Island, NY) was used to assess cell viability, according to the manufacturer's

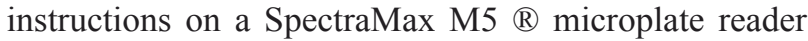
(Molecular Devices, Sunnyvale, CA).

Caspase-Glo 3/7 assay (Promega, UK) was used to measure caspase activity after 48 hours of treatment according to the manufacturer's instruction.

For cell cycle analysis, cells were treated for 12,24 and 48 hours. They were harvested, fixed with cold $70 \%$ ethanol for 30 minutes at $4^{\circ} \mathrm{C}$, and then incubated in the dark with RNase $(100 \mathrm{Mg} / \mathrm{ml})$ and propidium iodide (50 $\mathrm{Mg} / \mathrm{ml}$ ) for 30 minutes at $37^{\circ} \mathrm{C}$. A total of 20,000 cells were examined by flow cytometry using a Canto I flow cytometer (Becton-Dickinson, Franklin Lakes, NJ). Data were analyzed using ModFit software (Verity Software House, Topsham, ME).

\section{Migration assay}

Cell migration was assessed using a Transwell chamber assay (BD Biosciences, San Jose, CA) according to the manufacturer's protocol. The lower chamber of the plate was filled with $750 \mu \mathrm{L}$ DMEM supplemented with $10 \%$ FBS as a chemoattractant. After 22 hours of incubation at $37^{\circ} \mathrm{C}$, the cells invading through the bottom surface of the inserts were fixed, stained with Diff-Quik (Dade Behring Newark, NJ), and photographed and counted using Image J software (NIH, Bethesda, MD).

\section{Western blot and antibodies}

Total cell lysates were analyzed by SDSPAGE, transferred to a nitrocellulose membrane, and immunostained with the following antibodies overnight at $4^{\circ} \mathrm{C}$ : anti-vimentin $(1: 1000$, ab92547, AbCam, Cambridge, MA); anti-CD44 (1:1000, \#5640, Cell Signaling Technology, Beverly, MA); anti-p-AKT Ser473 (1:1000, \#9271, Cell Signaling Technology); anti-AKT (1:1000, \#9272, Cell Signaling Technology); anti-p-mTOR Ser2448 (1:1000, \#2971, Cell Signaling Technology); anti-mTOR (1:1000, \#2972, Cell Signaling Technology); anti-pp70S6K Thr421/Ser424 (1:1000, \#9204, Cell Signaling Technology); anti-70S6K (1:1000,\#2708, Cell Signaling
Technology); anti-p-4E-BP1 Thr37/46 (1:1000, \#2855, Cell Signaling Technology); anti-4E-BP1 (1:1000, \#9452, Cell Signaling Technology); anti-p-PRAS40 (1:1000, \#13175, Cell Signaling Technology), anti-PRAS40 (1:1000, \#2691, Cell Signaling Technology), anti-Survivin (1:1000, \#2808, Cell Signaling Technology), anti-Ncadherin (1:1000, \#04-1126, EMD Millipore, Billerica, MA); and anti-human GAPDH (1:5000, sc-32233, Santa Cruz Biotechnology, Santa Cruz, CA) and anti-beta actin (1:5000, sc-81178, Santa Cruz Biotechnology) were used as loading controls. The membranes were incubated with the appropriate horseradish peroxidase-conjugated IgG (anti-rabbit 1:3000, Cell Signaling Technology, or antimouse 1:10000, Santa Cruz Biotechnology), and proteins were detected by enhanced chemiluminescence (ECL; Thermo scientific, Rockford, IL).

\section{Apoptosis array}

The expression profile of apoptosis-related proteins was detected and analyzed using a human apoptosis array kit (ARY009), according to the manufacturer's instruction (R\&D Systems, Minneapolis, MN). The membranes containing apoptosis-related antibodies were blocked with Array buffer for $1 \mathrm{~h}$ on a rocking platform and then incubated with lysates of untreated (DMSO) or Torin2 treated ATC cell lines (8505c, C643 and SW1736) overnight at $4^{\circ} \mathrm{C}$. Chemiluminescent detection reagent was used after incubation with streptavidinHRP conjugate. The membranes were scanned and pixel density was quantified by the mean of two replicate spot densities normalized to internal background using Image J software. Targets showing a two-fold difference in density to background ratio (increase or decrease) when compared to vehicle were selected.

\section{Immunohistochemistry}

Tissue microarrays were purchased from US Biomax, Rockville, MD (\#TH641) and included duplicate samples of 6 follicular adenomas, 6 follicular thyroid carcinomas, 6 papillary thyroid carcinomas, and 6 ATC, and 6 normal tissues from thyroid, 10 from lungs, testis, and adrenal pheochromocytoma. Slides were deparaffinized and rehydrated, and incubated with the primary antibody for phospho-mTOR Ser2448 and mTOR (Cell Signaling Technology) overnight as previously reported [45]. Staining intensity and percent of positive cells were analyzed and scored blinded by a clinical pathologist (P.K.). Further, mouse lung tissue slides from in vivo mouse studies were deparaffinized, rehydrated, and incubated with the primary antibody for phospho-4EBP1 Thr37/46 (Cell Signaling Technology). The extent of staining (percentage of positive cell: 10/high power field) and intensity ( 0 for negative, 1 for weakly positive, 
2 for moderately positive and 3 for strongly positive) were determined and an $\mathrm{H}$ score was assigned to each tissue section as a product of the extent of immunoexpression and the intensity of staining as previously described [45]. Additionally, anti-Survivin (NB500-201, Novusbio, Littleton, CO) at 1:500 dilution, and anti-HIF-1 $\alpha$ ( [ESEE122,], ab8366, Abcam, Cambridge, MA) at 1:500 dilution were used for immunostaining using Vectastain $\mathrm{ABC}$ and DAB kits (Vector Laboratories, Inc. Burlingame, CA). Slides were scanned at cited magnification using a ScanScope XT digital slide scanner and viewed using ImageScope software (Aperio Technologies, Inc., Vista, CA).

\section{In vivo ATC metastasis mouse model}

An in vivo ATC metastasis mouse model was used to assess the effect of Torin 2 on growth and metastasis as previously described [19]. 8505c cells, stably transfected with a luciferase reporter gene $L u c 2$, were injected into the tail vein of six- to eight-week-old NOD.Cg-Prkdcscid Il $2 \mathrm{rgtm} 1 \mathrm{Wjl} / \mathrm{SzJ}$ mice purchased from The Jackson Laboratory (Maine, USA). The mice were maintained and bred according to the guidelines of the institute's Animal Advisory Committee. Immediately after tail vein injection, bioluminescence imaging was used to assess the injected cells in all mice using the Xenogen in vivo imaging system (Caliper Life Sciences Inc., Hopkinton, MA). Mice were injected intraperitoneally with $30 \mathrm{mg} / \mathrm{mL}$ of luciferin 15 minutes prior to imaging. Signal intensity was quantified as the sum of all detected photon counts within a region of interest using IVIS Living Image software (Caliper Life Sciences Inc., Hopkinton, MA).

To confirm tumor development in specific organ sites, ex vivo images were obtained. After whole body imaging, the mice were euthanized by $\mathrm{CO} 2$ inhalation and the organs were isolated and imaged again with the Xenogen system. Then the organs were fixed in formalin for histologic analysis. For animal survival analysis, mice were maintained under normal immunodeficient mouse care conditions, and monitored daily to assess their health status. Once the mice reached the humane euthanasia endpoints they were euthanized. Before euthanization, mice were imaged as described above.

\section{Statistical analyses}

qHTS curve fitting and data analysis were performed as previously described [46]. Statistical analyses were performed using GraphPad Prism 5 software (GraphPad Software, La Jolla, CA). Parametric and nonparametric data were analyzed using a two-tailed test and the Mann-Whitney U test, respectively. Survival analysis was performed using the Mantel Cox log rank test. $P<0.05$ was considered statistically significant. Data are presented as mean \pm standard deviation (SD) or mean \pm standard error of mean (SEM).

\section{FINANCIAL SUPPORT}

This research was supported by the Intramural Research Program of the Center for Cancer Research, National Cancer Institute, National Institutes of Health.

\section{DISCLOSURE OF POTENTIAL CONFLICTS OF INTERESTS}

The authors have no conflicts of interests to disclose.

\section{REFERENCES}

1. Davies L and Welch HG. Increasing incidence of thyroid cancer in the United States, 1973-2002. JAMA : the journal of the American Medical Association. 2006; 295:21642167.

2. Nilubol N and Kebebew E. Should small papillary thyroid cancer be observed? A population-based study. Cancer. 2014.

3. Smallridge $\mathrm{RC}$, Ain $\mathrm{KB}$, Asa SL, Bible $\mathrm{KC}$, Brierley JD, Burman KD, Kebebew E, Lee NY, Nikiforov YE, Rosenthal MS, Shah MH, Shaha AR, Tuttle RM and American Thyroid Association Anaplastic Thyroid Cancer Guidelines T. American Thyroid Association guidelines for management of patients with anaplastic thyroid cancer. Thyroid : official journal of the American Thyroid Association. 2012; 22:1104-1139.

4. Kebebew E, Greenspan FS, Clark OH, Woeber KA and McMillan A. Anaplastic thyroid carcinoma. Treatment outcome and prognostic factors. Cancer. 2005; 103:13301335.

5. Kebebew E. Anaplastic thyroid cancer: rare, fatal, and neglected. Surgery. 2012; 152:1088-1089.

6. DiMasi JA, Hansen RW and Grabowski HG. The price of innovation: new estimates of drug development costs. J Health Econ. 2003; 22:151-185.

7. Nilubol N, Zhang L, Shen M, Zhang YQ, He M, Austin $\mathrm{CP}$ and Kebebew E. Four clinically utilized drugs were identified and validated for treatment of adrenocortical cancer using quantitative high-throughput screening. Journal of translational medicine. 2012; 10:198.

8. Huang R, Southall N, Wang Y, Yasgar A, Shinn P, Jadhav A, Nguyen DT and Austin CP. The NCGC pharmaceutical collection: a comprehensive resource of clinically approved drugs enabling repurposing and chemical genomics. Sci Transl Med. 2011; 3:80ps16.

9. Zhang L, He M, Zhang Y, Nilubol N, Shen M and Kebebew E. Quantitative high-throughput drug screening identifies novel classes of drugs with anticancer activity in thyroid cancer cells: opportunities for repurposing. The Journal of 
clinical endocrinology and metabolism. 2012; 97:E319-328.

10. Shimaoka K, Schoenfeld DA, DeWys WD, Creech RH and DeConti R. A randomized trial of doxorubicin versus doxorubicin plus cisplatin in patients with advanced thyroid carcinoma. Cancer. 1985; 56:2155-2160.

11. Higashiyama T, Ito Y, Hirokawa M, Fukushima M, Uruno T, Miya A, Matsuzuka F and Miyauchi A. Induction chemotherapy with weekly paclitaxel administration for anaplastic thyroid carcinoma. Thyroid : official journal of the American Thyroid Association. 2010; 20:7-14.

12. Denaro N, Nigro CL, Russi EG and Merlano MC. The role of chemotherapy and latest emerging target therapies in anaplastic thyroid cancer. OncoTargets and therapy. 2013; 9:1231-1241.

13. Kracikova M, Akiri G, George A, Sachidanandam R and Aaronson SA. A threshold mechanism mediates p53 cell fate decision between growth arrest and apoptosis. Cell death and differentiation. 2013; 20:576-588.

14. Gulhati P, Bowen KA, Liu J, Stevens PD, Rychahou PG, Chen M, Lee EY, Weiss HL, O'Connor KL, Gao T and Evers BM. mTORC1 and $\mathrm{mTORC} 2$ regulate EMT, motility, and metastasis of colorectal cancer via RhoA and Rac1 signaling pathways. Cancer research. 2011; 71:3246-3256.

15. Liu J and Brown RE. Immunohistochemical detection of epithelialmesenchymal transition associated with stemness phenotype in anaplastic thyroid carcinoma. International journal of clinical and experimental pathology. 2010; 3:755762.

16. Ma XM and Blenis J. Molecular mechanisms of mTORmediated translational control. Nature reviews Molecular cell biology. 2009; 10:307-318.

17. She QB, Halilovic E, Ye Q, Zhen W, Shirasawa S, Sasazuki T, Solit DB and Rosen N. 4E-BP1 is a key effector of the oncogenic activation of the AKT and ERK signaling pathways that integrates their function in tumors. Cancer cell. 2010; 18:39-51.

18. Madhunapantula SV, Sharma A and Robertson GP. PRAS40 deregulates apoptosis in malignant melanoma. Cancer research. 2007; 67:3626-3636.

19. Zhang L, Gaskins K, Yu Z, Xiong Y, Merino MJ and Kebebew E. An in vivo mouse model of metastatic human thyroid cancer. Thyroid : official journal of the American Thyroid Association. 2014; 24:695-704.

20. Pannone G, Santoro A, Pasquali D, Zamparese R, Mattoni M, Russo G, Landriscina M, Piscazzi A, Toti P, Cignarelli M, Lo Muzio L and Bufo P. The role of survivin in thyroid tumors: differences of expression in well-differentiated, non-well-differentiated, and anaplastic thyroid cancers. Thyroid : official journal of the American Thyroid Association. 2014; 24:511-519.

21. Sarela AI, Macadam RC, Farmery SM, Markham AF and Guillou PJ. Expression of the antiapoptosis gene, survivin, predicts death from recurrent colorectal carcinoma. Gut. 2000; 46:645-650.
22. Harris AL. Hypoxia--a key regulatory factor in tumour growth. Nature reviews Cancer. 2002; 2:38-47.

23. Wang N, Dong CR, Jiang R, Tang C, Yang L, Jiang QF, Chen GG and Liu ZM. Overexpression of HIFlalpha, metallothionein and SLUG is associated with high TNM stage and lymph node metastasis in papillary thyroid carcinoma. International journal of clinical and experimental pathology. 2014; 7:322-330.

24. Courtney KD, Corcoran RB and Engelman JA. The PI3K pathway as drug target in human cancer. Journal of clinical oncology : official journal of the American Society of Clinical Oncology. 2010; 28:1075-1083.

25. Anandappa G, Hollingdale A and Eisen T. Everolimus a new approach in the treatment of renal cell carcinoma. Cancer management and research. 2010; 2:61-70.

26. Zhang YJ, Duan Y and Zheng XF. Targeting the mTOR kinase domain: the second generation of mTOR inhibitors. Drug discovery today. 2011; 16:325-331.

27. Papewalis C, Wuttke M, Schinner S, Willenberg HS, Baran AM, Scherbaum WA and Schott M. Role of the novel mTOR inhibitor RAD001 (everolimus) in anaplastic thyroid cancer. Hormone and metabolic research $=$ Hormon- und Stoffwechselforschung $=$ Hormones et metabolisme. 2009; 41:752-756.

28. Liu J and Brown RE. Morphoproteomics demonstrates activation of mTOR pathway in anaplastic thyroid carcinoma: a preliminary observation. Annals of clinical and laboratory science. 2010; 40:211-217.

29. Rini BI and Atkins MB. Resistance to targeted therapy in renal-cell carcinoma. The lancet oncology. 2009; 10:9921000 .

30. Wan X, Harkavy B, Shen N, Grohar P and Helman LJ. Rapamycin induces feedback activation of Akt signaling through an IGF-1R-dependent mechanism. Oncogene. 2007; 26:1932-1940.

31. Zhang H, Berel D, Wang Y, Li P, Bhowmick NA, Figlin RA and Kim HL. A comparison of Ku0063794, a dual mTORC1 and mTORC2 inhibitor, and temsirolimus in preclinical renal cell carcinoma models. PloS one. 2013; 8:e54918.

32. Liu Q, Xu C, Kirubakaran S, Zhang X, Hur W, Liu Y, Kwiatkowski NP, Wang J, Westover KD, Gao P, Ercan D, Niepel M, Thoreen CC, Kang SA, Patricelli MP, Wang $\mathrm{Y}$, et al. Characterization of Torin2, an ATP-competitive inhibitor of mTOR, ATM, and ATR. Cancer research. 2013; 73:2574-2586.

33. Simioni C, Cani A, Martelli AM, Zauli G, Tabellini G, McCubrey J, Capitani S and Neri LM. Activity of the novel mTOR inhibitor Torin-2 in B-precursor acute lymphoblastic leukemia and its therapeutic potential to prevent Akt reactivation. Oncotarget. 2014; 5:10034-10047.

34. Ahmed M, Hussain AR, Bavi P, Ahmed SO, Alsobhi SS, Al-Dayel F, Uddin S and Al-Kuraya KS. High prevalence of mTOR complex activity can be targeted using Torin 2 in 
papillary thyroid carcinoma. Carcinogenesis. 2014.

35. Wagle N, Grabiner BC, Van Allen EM, Amin-Mansour A, Taylor-Weiner A, Rosenberg M, Gray N, Barletta JA, Guo Y, Swanson SJ, Ruan DT, Hanna GJ, Haddad RI, Getz G, Kwiatkowski DJ, Carter SL, et al. Response and acquired resistance to everolimus in anaplastic thyroid cancer. The New England journal of medicine. 2014; 371:1426-1433.

36. Petermann E, Helleday $\mathrm{T}$ and Caldecott KW. Claspin promotes normal replication fork rates in human cells. Molecular biology of the cell. 2008; 19:2373-2378.

37. Ito Y, Yoshida H, Uruno T, Nakano K, Miya A, Kobayashi K, Yokozawa T, Matsuzuka F, Matsuura N, Kakudo $\mathrm{K}$, Kuma K and Miyauchi A. Survivin expression is significantly linked to the dedifferentiation of thyroid carcinoma. Oncology reports. 2003; 10:1337-1340.

38. Smallridge RC, Marlow LA and Copland JA. Anaplastic thyroid cancer: molecular pathogenesis and emerging therapies. Endocrine-related cancer. 2009; 16:17-44.

39. Pita JM, Figueiredo IF, Moura MM, Leite V and Cavaco BM. Cell cycle deregulation and TP53 and RAS mutations are major events in poorly differentiated and undifferentiated thyroid carcinomas. The Journal of clinical endocrinology and metabolism. 2014; 99:E497-507.

40. Garcia-Rostan G, Costa AM, Pereira-Castro I, Salvatore G, Hernandez R, Hermsem MJ, Herrero A, Fusco A, Cameselle-Teijeiro $\mathrm{J}$ and Santoro M. Mutation of the PIK3CA gene in anaplastic thyroid cancer. Cancer research. 2005; 65:10199-10207.

41. Nehs MA, Nagarkatti S, Nucera C, Hodin RA and Parangi S. Thyroidectomy with neoadjuvant PLX4720 extends survival and decreases tumor burden in an orthotopic mouse model of anaplastic thyroid cancer. Surgery. 2010; 148:1154-1162; discussion 1162.

42. Nucera C, Nehs MA, Mekel M, Zhang X, Hodin R, Lawler J, Nose V and Parangi S. A novel orthotopic mouse model of human anaplastic thyroid carcinoma. Thyroid : official journal of the American Thyroid Association. 2009; 19:1077-1084.

43. Nucera C, Porrello A, Antonello ZA, Mekel M, Nehs MA, Giordano TJ, Gerald D, Benjamin LE, Priolo C, Puxeddu E, Finn S, Jarzab B, Hodin RA, Pontecorvi A, Nose V, Lawler $\mathrm{J}$, et al. B-Raf(V600E) and thrombospondin-1 promote thyroid cancer progression. Proceedings of the National Academy of Sciences of the United States of America. 2010; 107:10649-10654.

44. Marlow LA, D’Innocenzi J, Zhang Y, Rohl SD, Cooper SJ, Sebo T, Grant C, McIver B, Kasperbauer JL, Wadsworth JT, Casler JD, Kennedy PW, Highsmith WE, Clark O, Milosevic D, Netzel B, et al. Detailed molecular fingerprinting of four new anaplastic thyroid carcinoma cell lines and their use for verification of RhoB as a molecular therapeutic target. The Journal of clinical endocrinology and metabolism. 2010; 95:5338-5347.

45. Ghayee HK, Giubellino A, Click A, Kapur P, Christie A,
Xie XJ, Martucci V, Shay JW, Souza RF and Pacak K. Phospho-mTOR is not upregulated in metastatic SDHB paragangliomas. European journal of clinical investigation. 2013; 43:970-977.

46. Inglese J, Auld DS, Jadhav A, Johnson RL, Simeonov A, Yasgar A, Zheng W and Austin CP. Quantitative highthroughput screening: a titration-based approach that efficiently identifies biological activities in large chemical libraries. Proceedings of the National Academy of Sciences of the United States of America. 2006; 103:11473-11478. 